\title{
Investigating the epi-miRNome: identification of epi-miRNAs using transfection experiments
}

\author{
Elisa Reale ${ }^{*, \dagger}, 1$, Daniela Taverna ${ }^{\dagger, 2,3,4}$, Laura Cantini ${ }^{56}$, Loredana Martignetti ${ }^{5}$, Matteo

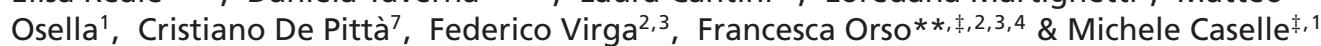 \\ ${ }^{1}$ Department of Physics \& INFN, University of Torino, 10125, Torino, Italy \\ ${ }^{2}$ Molecular Biotechnology Center (MBC), 10126, Torino, Italy \\ ${ }^{3}$ Department of Molecular Biotechnology \& Health Sciences, 10126, Torino, Italy \\ ${ }^{4}$ Center for Complex Systems in Molecular Biology \& Medicine, University of Torino, 10123, Torino, Italy \\ ${ }^{5}$ Institut Curie, PSL Research University, INSERM U900, Paris, France \\ ${ }^{6}$ Computational Systems Biology Team, Institut de Biologie de I'Ecole Normale Supérieure, CNRS UMR8197, INSERM U1024, Ecole \\ Normale Supérieure, Paris Sciences et Lettres Research University, 75005 Paris, France \\ ${ }^{7}$ Department of Biology, University of Padova, 3522, Padova, Italy \\ *Author for correspondence: Tel.: +39 0116707205 ; elisa.reale@unito.it \\ **Author for correspondence: Tel.: +39 011670 6448; Fax: +39 011670 6432; francesca.orso@unito.it \\ $\dagger$ Authors contributed equally as first coauthors \\ $¥$ Authors contributed equally as last coauthors
}

\begin{abstract}
Aim: Growing evidence shows a strong interplay between post-transcriptional regulation, mediated by miRNAs (miRs) and epigenetic regulation. Nevertheless, the number of experimentally validated miRs (called epi-miRs) involved in these regulatory circuitries is still very small. Material \& methods: We propose a pipeline to prioritize candidate epi-miRs and to identify potential epigenetic interactors of any given miR starting from miR transfection experiment datasets. Results \& conclusion: We identified 34 candidate epi-miRs: 19 of them are known epi-miRs, while 15 are new. Moreover, using an in-house generated gene expression dataset, we experimentally proved that a component of the polycomb-repressive complex 2 , the histone methyltransferase enhancer of zeste homolog 2 (EZH2), interacts with miR-214, a well-known prometastatic miR in melanoma and breast cancer, highlighting a miR-214-EZH2 regulatory axis potentially relevant in tumor progression.
\end{abstract}

First draft submitted: 19 February 2019; Accepted for publication: 5 September 2019; Published online: 6 November 2019

Keywords: epi-miRNA • epigenetic • EZH2 • feedback loop • miR-138 • miR-145 • miR-214 • miR-31 • miRs • polycomb complex $\bullet$ tumor progression

miRNAs (miRs) are small noncoding RNAs that negatively regulate gene expression in metazoans and plants [1,2]. They are relevant in physiological processes ranging from development and metabolism to apoptosis and signaling pathways [3,4]. Moreover, their expression is altered in several human diseases, particularly in cancer [5,6], making miRs a major focus of research in molecular biology.

In the last few years, the interplay between miRs and the epigenetic layers of regulation has been intensely investigated. A growing amount of evidence supports the importance of a few selected miRs in regulating epigenetic factors [7]. At the same time, several miRs (and among them most of the known epi-miRs) have been shown to be under strict epigenetic control [7-11]. This interplay is so deep that it has been recently suggested that epigenetic regulation could be only understood considering epigenetic regulators (epi-Rs) and miRs as partners of combined regulatory circuitries [12]. Four are the main epigenetic pathways involved in this kind of networks and considered in our analysis. The polycomb-repressive complex 1 (PRC1), a transcriptional-repressor complex consisting of several proteins among which an important role is played by the Polycomb group ring finger ones (in particular Bmi-1) and whose action is responsible of the ubiquitylation of H2AK119ub1 [13]. Belonging to the same family of repressors, the PRC2 is composed of four subunits: RbAp48, SUZ12, the histone methyltransferase EZH2 and 
EED. PRC2 can work alone by adding the repressive mark of trimethylated histone H3 at lysine 27 (H3K27me3) to its target genes or in cooperation with NIPP1 [14] to increase its repressive action. DNA methyltransferases (DNMT) involved in DNA methylation have, in general, a transcriptional-repressive role, especially on CpG-rich promoters [15]; while histone deacetylases (HDACs), involved in histone deacetylation, act mainly as transcriptional corepressors [16]. In order to simplify the analysis, we made the assumption that these four epigenetic pathways exert their function mainly as repressors even if, for example, HDACs can also act as activators in some specific cases [17]. All the mentioned epigenetic pathways can interact. For instance, there is evidence of an interaction between HDAC and PRC1 (and possibly PRC2) [18]. Notwithstanding their importance, the number of experimentally validated miRs involved in regulatory circuitries with the above-mentioned epi-Rs, from here on referred to as epi-miRs, is still very small, their identification episodic, and no attempt was performed up to now for a systematic search. The main goal of this paper is to fill this gap. We propose a simple protocol to identify candidate epi-miRs and study their mechanism of action combining gene expression datasets coming from experiments of overexpression of a given miR and data of knockdown, knockout, drug-mediated downregulation or chromatin immunoprecipitation (ChIP) experiments of a given epi- $\mathrm{R}$ (from here on referred to as epi-R ablation).

Moreover, our procedure can also be used to identify candidate epigenetic interactors of a given miR of interest and as an example we studied the case of miR-214, a prometastatic miR $[19,20]$ involved in the coordination of melanoma tumor cell migration, invasion, adhesion to extracellular matrices, transendothelial migration and survival to anoikis in vitro, as well as extravasation from blood vessels and metastasis formation in vivo [19]. miR-214 acts through the simultaneous coordination of a network including over 70 protein-coding genes and the antimetastatic small noncoding RNA, miR-148b [20]. In the present work, we experimentally proved that the EZH2, the main component of the PRC2, is an interactor of miR-214, involved, at least partially, in the activation of miR-214 downstream players: ITGA5, ALCAM and the small noncoding RNA, miR-148b. Due to the importance of EZH2 as a crucial epi-R involved in the initiation and advancement of melanoma [21], the described miR-214-EZH2 axis could be of potential interest to understand tumor progression.

\section{Methods}

\section{GSEA gene sets}

Gene sets were downloaded from the MSigDB database (v5.2 updated October 2016) [22,23], which contains gene sets collected from various sources, from on-line pathway databases to PubMed publications. We selected a panel of gene sets broadly related to epigenetic pathways as discussed in the main text. We chose, when possible, multiple sets for a specific component, spanning over different tissues or experimental conditions. We ended up with a panel of 43 sets, summarized in Supplementary File 1. These sets can be divided into two main classes: results of ChIP experiments, whose entries allow to directly identify putative targets of the selected factor; results of gene expression experiments after knockdown, knockout or drug-mediated downregulation a particular epi-R. In this case, we downloaded separately both the upregulated and the downregulated genes. These allow us to fix also the sign and in some cases the direction of the regulatory interactions we found.

\section{miR transfection experiment datasets}

Fourteen series of miR transfection experiments on five different platforms by Seki et al. [24-38], for a total of 157 gene expression datasets involving 59 miRs transfected in different human cancer cell lines were downloaded from GEO. Gene expression data were obtained using Agilent whole-genome microarrays. An experiment series from Misiewicz-Krzeminska et al. [39], containing data from miR-214 expression in H929 myeloma cell lines was also downloaded to support our experimental data. The data were LOWESS normalized and background subtracted from $\log 10$ of processed Red signal/processed Green signal by the author through Agilent software (Supplementary File 2). Some of the gene names annotated to the probes were obsolete and we updated them, exploiting the bioconductoR (Bioconductor 3.2, R 3.2.3) package biomaRt (v. 2.34.2) [40]. Then, we selected the probes with the highest fold change across each platform, we selected a unique set of genes conform to all the experiments considered to use as universe gene set (containing 17,025 genes) and applied an arbitrary, the literature-approved, threshold of $\mathrm{t}=0.5$ on the $\log 10$ (fold change) of the expression data to select the up-/downregulated genes for each experiment in the Seki panel and in the GSE35948-miR-214 panel. 


\section{miRTarBase targets}

The miRTarBase database [41] was used to identify putative targets broadly related to the epigenetic pathways we were interested in. We differentiated among weak (validated by CLIP-seq) or strong (validated using reporter assays or western blots) miR-target interactions by the database annotation.

\section{TargetScan analysis}

TargetScan (http://www.targetscan.org/vert_72/) [42] was used to compare our results with those expected by a canonical miR-target direct-repressive interaction. We downloaded the files for conserved and not conserved miRfamilies and sites of interaction (TargetScan release 7.2, March 2018) and we computed the number of predicted targets with no regard for the conservation or the score given to a specific site. To avoid repetitions, we performed the statistical analysis only on those miRs that resulted miR or candidate epi-miRs in all the transfection experiments.

\section{Reagents \& antibodies}

Pre-miR miRNA Precursor Molecules Negative Control 1, miRNA precursors hsa-miR-214 (PM12124; Ambion, TX, USA). TaqMan miRNA assays for miRNA detection: hsa-miR-148b (000471) and U6 snRNA (001973) (Applied Biosystems, CA, USA). siRNAs: siEZH2 (Hs-EZH2- FlexiTube siRNA SI02665166) and All Stars Negative Control siRNA were purchased from Qiagen (CA, USA). Primary antibodies: anti-actin pAb I-19 and anti-GAPDH pAb V-18 were from Santa Cruz Biotechnology (TX, USA); anti-ITGA5 pAb RM10 was kindly provided by G Tarone (University of Torino, Torino, Italy); anti-EZH2 mAb \#612666 (BD, Transduction Laboratories, CA, USA); anti-CD166/ALCAM mAb MOG/07 (Novocastra Laboratories, Newcastle Upon Tyne, UK). Secondary antibodies: goat antimouse and goat antirabbit HRP-conjugated IgG were from Santa Cruz Biotechnology. All antibodies were used at the producer's suggested concentrations. Real-Time assays: QuantiTect Primer Assay (200) Hs-GAPDH-1-SG QuantiTect Primer Assay QT00079247, QuantiTect Primer Assay (200) Hs-ALCAM-1-SG QuantiTect Primer Assay QT00026824; QuantiTect Primer Assay (200) Hs-ITGA5-1- SG QuantiTect Primer Assay QT00080871 (Qiagen, CA, USA).

\section{Cell culture}

MA-2 cells were provided by R.O. Hynes (Massachusetts Institute of Technology, MA, USA) and maintained as described previously [19,20].

\section{Transient transfections of pre-miRs \& siRNAs}

To obtain transient pre-miR or siRNA expression, cells were transfected using RNAiFect (Qiagen, CA, USA) reagent, $75 \mathrm{nM}$ pre-miR and $170 \mathrm{nM}$ siRNA, as described in [19].

\section{RNA isolation \& quantitative Reverse Transcription (qRT)-PCR for miRNA or mRNA Detection}

Total RNA was isolated from cells using TRIzol Reagent (Invitrogen Life Technologies, CA, USA) according to manufacturers' protocol. qRT-PCRs were performed as described in [19].

\section{Gene expression profiling}

Protein-coding expression profiling was carried out using the Whole Human Genome Oligo Microarray (41,000, 60-mer oligonucleotide probes) from Agilent Technologies. Total RNA (800 ng) was labeled by the Agilent One-Color Microarray-Based Gene Expression protocol, according to the manufacturer's instructions. Slides were scanned on an Agilent microarray scanner. Analysis was performed as described in [43]. Gene expression data are available in the U.S. Center for Biotechnology Information Gene Expression Omnibus (GEO) database (GSE124965).

\section{Protein preparation \& western blotting}

Total protein preparation and western blot analysis were performed as described in [19].

\section{Statistical analyses for biological experiments}

Data are presented as mean standard deviation (SD) or as mean standard error of the mean (SEM), as indicated, and two-tailed Student's t-test was used for comparison, with, $\mathrm{p}<0.05$; $\mathrm{p}<0.01 ; \mathrm{p}<0.001$ considered to be statistically significant. - indicates a non-statistically-significant p-value. 


\section{Results}

\section{Bioinformatics workflow for the identification of candidate epi-miRs}

The interplay of miRs and epi-Rs in regulatory networks can lead to extremely complex patterns of gene expression. However, it should be possible to infer the most likely regulatory circuitries underlying specific expression patterns, at least in simple cases. In general, the interaction between a miR and a global epi-R could lead to an epigenetic 'amplification' of the miR action that may explain the episodic empirical observation of a large number of genes varying their expression in miR transfection experiments. We propose a simple, but powerful method to test if this amplification is really the signature of the action of an epigenetic amplification starting from gene expression data of $\mathrm{miR}$ overexpression experiments together with data obtained following epi- $\mathrm{R}$ ablation. The rationale behind our approach is that, since most of considered epigenetic pathways are repressors of gene expression, if they are controlled (i.e., downregulated) by a miR, then all the genes targeted by the epigenetic machinery should be upregulated when the miR is overexpressed. All cases in which no significant intersection was observed between the $\mathrm{miR}$ and the epi-R regulated genes (see Figure 1A) were not further considered in our analysis because we assumed that the two players did not act cooperatively. Regulatory circuitries were considered when we found significant intersections: between the downregulated genes following miR overexpression and the upregulated genes in epi- $\mathrm{R}$ ablation experiments (Figure 1B); between the downregulated genes following miR overexpression and the epi-R ablation (Figure 1C); between the upregulated genes observed in both conditions (miR overexpression and epi-R ablation, Figure 1D); when the intersections described in Figure 1C and D were identified for the same miR in the same epigenetic pathway (Figure 1E). Clearly, this is a simplified view. Undirect interactions and more complex regulatory networks and cascades can, in principle, lead to similar expression patterns. Therefore, the regulatory circuits in Figure 1 should be seen as the most basic interactions between miR and epi-miR compatible with the experimental expression patterns. For example, cases in which several genes are both upregulated after miR overexpression and downregulated after epi- $\mathrm{R}$ ablation cannot be explained by these simple circuitries and require additional regulatory players.

More in details, the analysis was organized in three steps as depicted in Figure 2. First, we identified a panel of gene sets which could be considered strongly associated to epigenetic regulation. To this end, we selected the gene sets available from the gene set enrichment analysis (GSEA) database (software.broadinstitute.org/gsea/) [22,23] coming from epi- $\mathrm{R}$ ablation experiments. The main assumption in our protocol is that the epigenetic regulation is so strong and widespread that several of the target genes do not depend on the cell lines used in the experiment and can be used as a benchmark of the activation of the epigenetic pathway of interest. We chose gene sets associated to each of the epigenetic pathways discussed above, identifying 43 gene sets (reported in Supplementary File 1). The representation of the different epi-Rs in the identified datasets is summarized in Figure 3A. Briefly, 20 of them are specifically devoted to the PRC2 and contained both manually curated lists and experiments looking for the histone mark H3K27me3 associated to the PRC2 activity; while 23 were devoted to the other three epigenetic pathways discussed above. The number of genes contained in the sets ranges from 11 to more than 900, the majority of them (around 60\%) contains up to 150 genes. Some of the sets have a strong overlap in their gene content and we use them as a cross-check of the procedure. As second step, we used the above panel to evaluate for any given miR transfection experiment the probability of the involvement of the $\mathrm{miR}$ in one (or more) epigenetic pathways under study. To this end, we evaluated the intersection between each of the 43 gene sets and the sets of genes upregulated and downregulated in the transfection experiments. The significance of this intersection was assessed by a hypergeometric test corrected for multiple testing using the Benjamini-Hochberg procedure in order to have a well-defined false discovery rate (FDR). We set a threshold of 0.001 for the FDR. All the entries below this threshold represent candidate associations between a miR and an epigenetic pathway, which we prioritize for further analysis. Finally, in the third step, we classified the significant intersections in the different type of circuitries described in Figure 1. This could be accomplished, thanks to the fact that some of the gene sets correspond to Chip-seq experiments of epigenetic factors and that in other cases we have both the set of genes upregulated and downregulated when silencing crucial components of a given epigenetic machinery.

\section{Identification of candidate epi-miRs}

In order to test our procedure, 14 experiment series (Supplementary File 2) for a total of $157 \mathrm{miR}$ transfection experiments (Supplementary File 3) involving 59 different miRs on 26 different cell lines were downloaded from Gene Expression Omnibus (GEO) database (https://www.ncbi.nlm.nih.gov/geo/). This particular panel of transfections was chosen among the hundreds of miR transfection experiments available in the GEO database, 


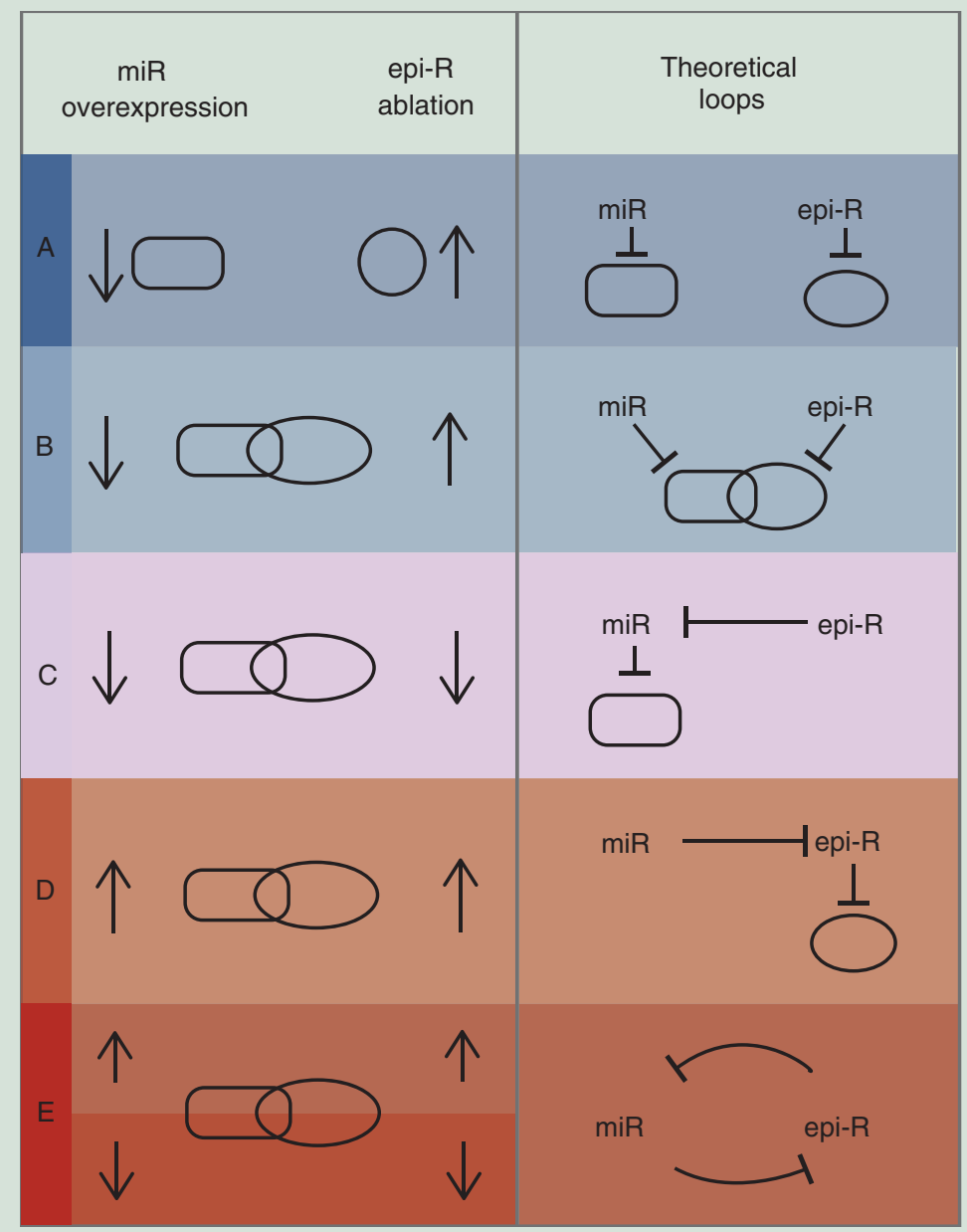

Figure 1. Schematic view of considered circuitries between miRNAs and epigenetic regulators. miR overexpression or epi-R ablation or chromatin immunoprecipitation experiments were used to identify the pattern of miR and epi-R interactions. The putative circuitries between miR and epi-R are depicted. In (A), miR and epi-R do not interact and regulate distinct sets of genes; in (B), they repress an overlapping set of genes; in (C), the epi-R represses the miR; in (D), the miR represses the epi-R; in (E), both (C) and (D) situations are present for the same miR and the same epigenetic pathway. The up arrows indicate the sets of genes upregulated in the depicted experiments, while the down arrows indicate the sets of downregulated genes. Cases in which the genes were upregulated after miR overexpression and downregulated after epi-R ablation were not took into consideration because it was not possible to group them under a single common theoretical circuit.

epi-R: Epigenetic regulator; miR: miRNA.

because they are characterized by a high level of homogeneity and consistency both of the experimental protocol and the data curation. Thirty-four out of 59 miRs resulted as candidate epi-miRs: 19 are known as epi-miRs, while 15 are identified as new, a pie chart showing the percentage of the different classes of miR/epi-miR is shown in Figure 3B. The results of the best intersections obtained for each candidate epi-miR and the associated epigenetic pathway are summarized in Table 1, where in the second column the association of each miR to the putative targeted epigenetic pathway is shown. In third, fourth, fifth and sixth columns, the potential loops between the candidate miR and epi-R belonging to the categories described in Figure 1 are indicated. Finally, in the last column, the results obtained using the miRTarBase database of experimentally validated miR-target interactions [41] are indicated (Supplementary File 4). Pathways displaying very strong signatures (FDR $<10 \times 10^{-8}$ ) are reported in bold face (complete list of intersections in Supplementary File 5). More than half (34) of the 59 miRs transfected in the analyzed panel of experiments turned out to be candidate epi-miRs. Notice, however, that this observation 


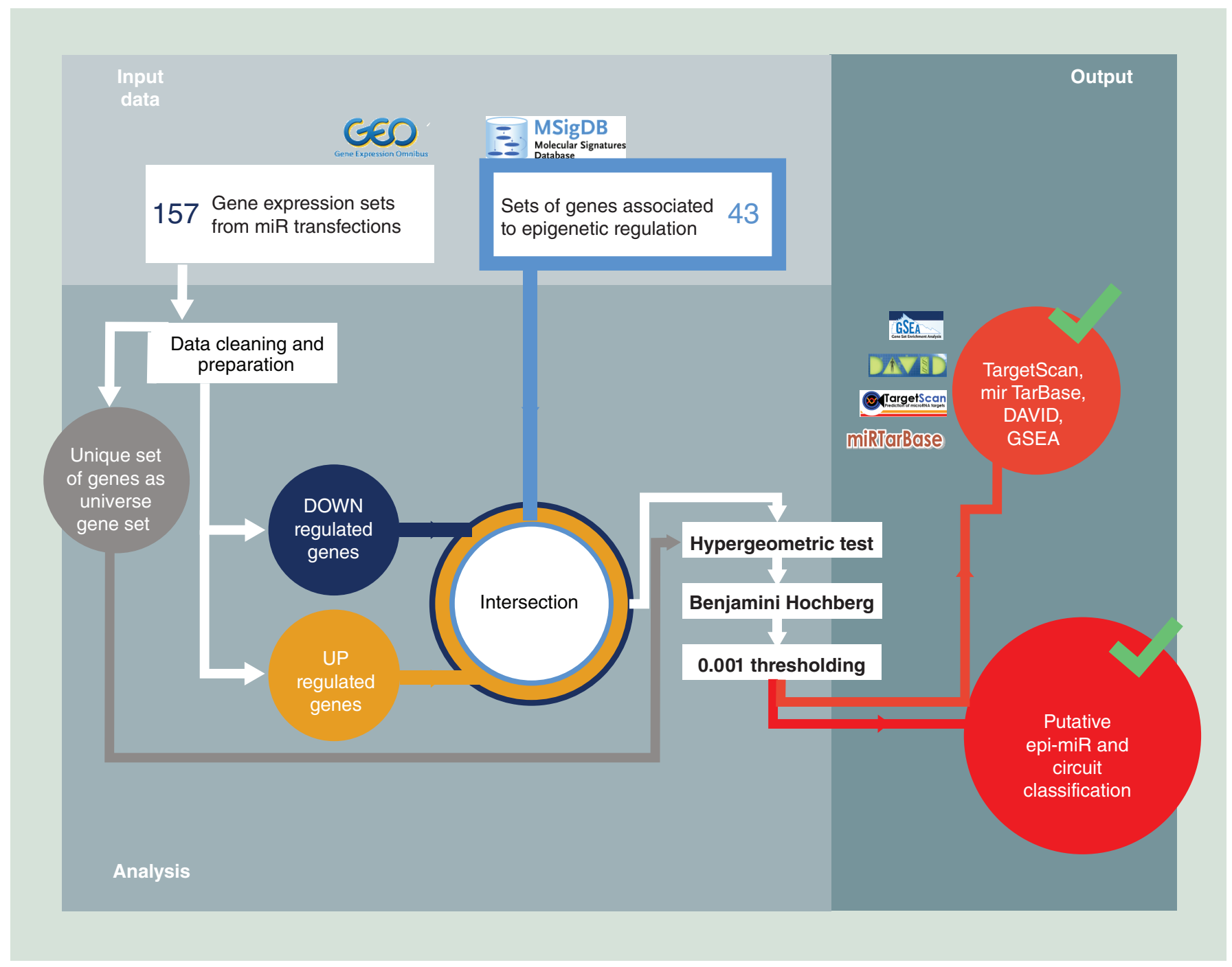

Figure 2. Schematic pipeline of our analysis. Each miRNA (miR) transfection experiment gene set was divided in up-/downregulated gene sets, and for each of them was computed the intersection with the mSigDb gene sets. The significance of these intersections has been evaluated through a hypergeometric test and corrected for multiple testing by the Benjamini-Hochberg procedure. All of the miRs involved in intersections with false discovery rate lower than 0.001 were selected as candidate experimentally validated miR. Results were further refined through gene set enrichment analysis, Database for Annotation, Visualization and Integrated Discovery, miRTarBase or TargetScan analysis, and each miR-epigenetic regulator interaction was labeled on a specific theoretical circuit based on the sign of the miR gene set and the type of epigenetic gene set involved in the intersection.

DAVID: Database for annotation, visualization and integrated discovery; epi-miR: Epigenetic miRNA; GSEA: Gene set enrichment analysis.

can be influenced by the fact that most of the miRs included in the panel are known to be involved in cancer or in differentiation processes and thus more likely to be involved in epigenetic pathways. In most of the cases (182 intersections corresponding to 55 different transfection experiments), we found very low FDR for the intersections of the upregulated genes with the GSEA sets, which could be compatible with a direct interaction of the miR with an epi-R. Interestingly, we also found quite a few examples (93 intersections corresponding to 38 different transfection experiments) of significant intersections for the downregulated genes. In a few cases, when transfection experiments of the same miR in different cell lines were available, we found that the same miR may act as an epi-miR in one particular cell line and show no epigenetic effect in the other cell lines.

The results showed a few clear correlations between the different epigenetic pathways analyzed. In general, for several miRs, we found signatures for more than one epigenetic pathway (23/34 epi-miRs, see Table 1$)$. In some cases, it is difficult to disentangle if these are due to a direct interaction of the miR with the epi- $\mathrm{R}$ or are triggered by the disregulation of other epigenetic pathways. 
(A)

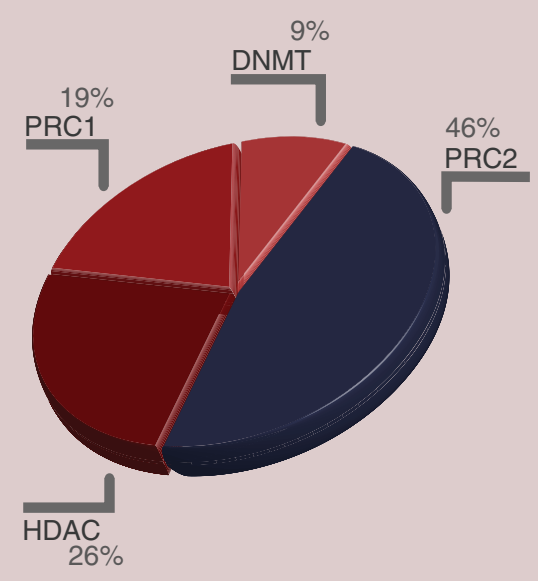

(c)

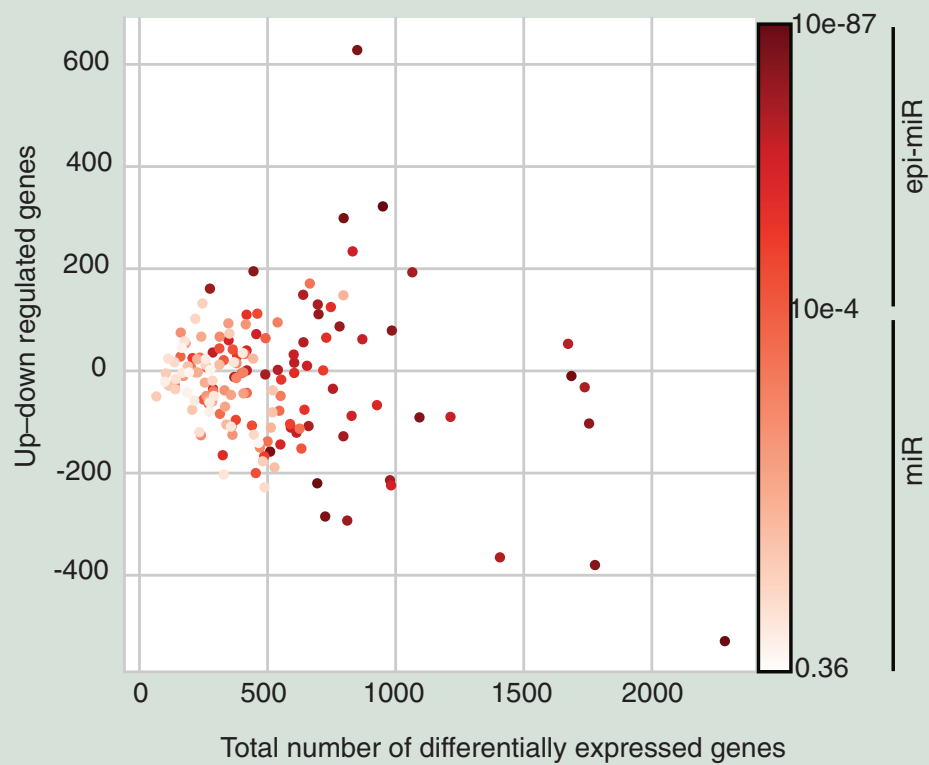

(B)

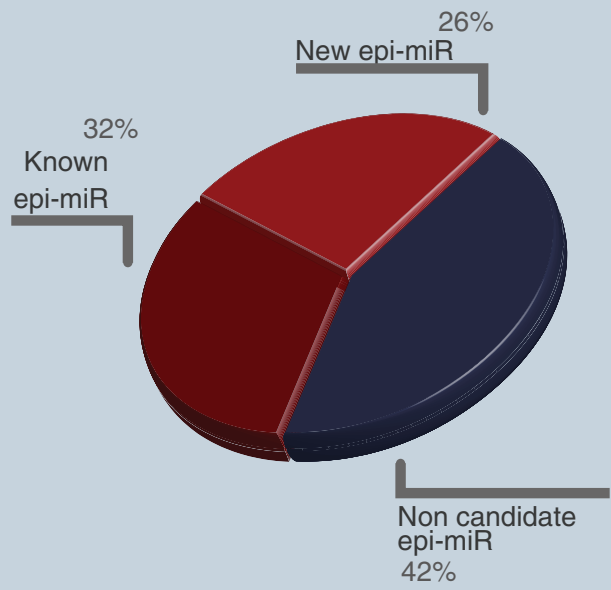

(D)

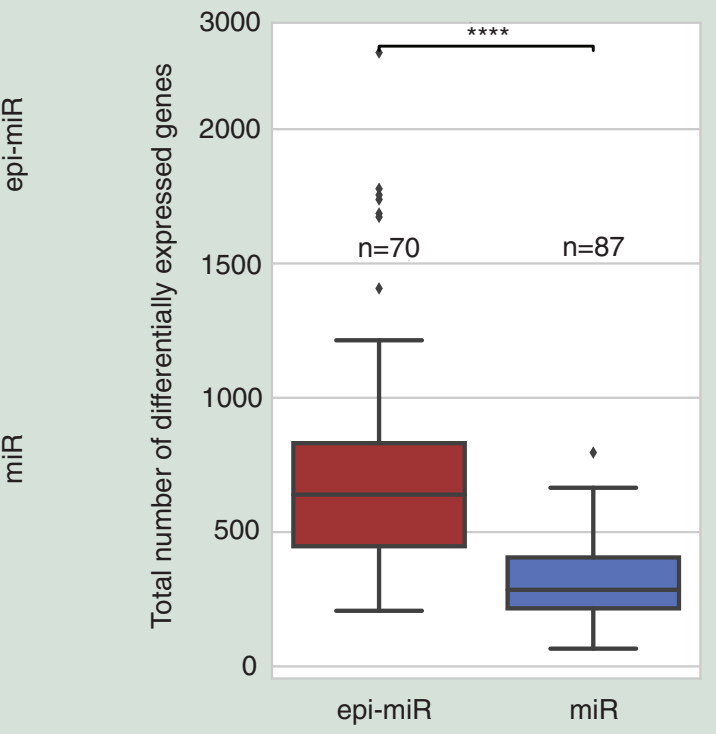

Figure 3. Candidate experimentally validated miRNAs show a large number of differentially expressed genes. (A) Percentage distribution of the mSigDb gene sets used among the epigenetic pathway taken into consideration. (B) Percentage distribution of candidate or not-candidate epi-miRs as resulted by our analysis. For those that resulted to be candidate epi-miRs, the percentages of new and known epi-miRs are reported. (C) Scatter plot showing the distribution of the difference among up- and downregulated genes (upregulated genes-downregulated genes) versus the total number of differentially expressed genes for all analyzed transfection experiments on the basis of the significance of their intersection with the gene set enrichment analysis sets. Each symbol represents one of the transfection experiments listed in Supplementary File 3. The epi-miR and miR identified by the gene set enrichment analysis intersection analysis are shown in red color gradation as indicated in the false discovery rate color bar. (D) Box plots representing the total number of differentially expressed genes for the indicated $(n)$ number of experiments, discriminating between those where the miRs turned out to behave as epi-miRs (red, left) and those where they acted as regular miRs (blue, right). In (C), false discovery rate was obtained using a Benjamini-Hochberg procedure. In (D), significance was assessed using a Mann-Whitney U two tailed test, $* * * * p$-value $<0.0001$; - a nonstatistically significant $p$-value. epi-miR: Experimentally validated miRNA. 


\section{Table 1. List of candidate epigenetic miRNAs.}

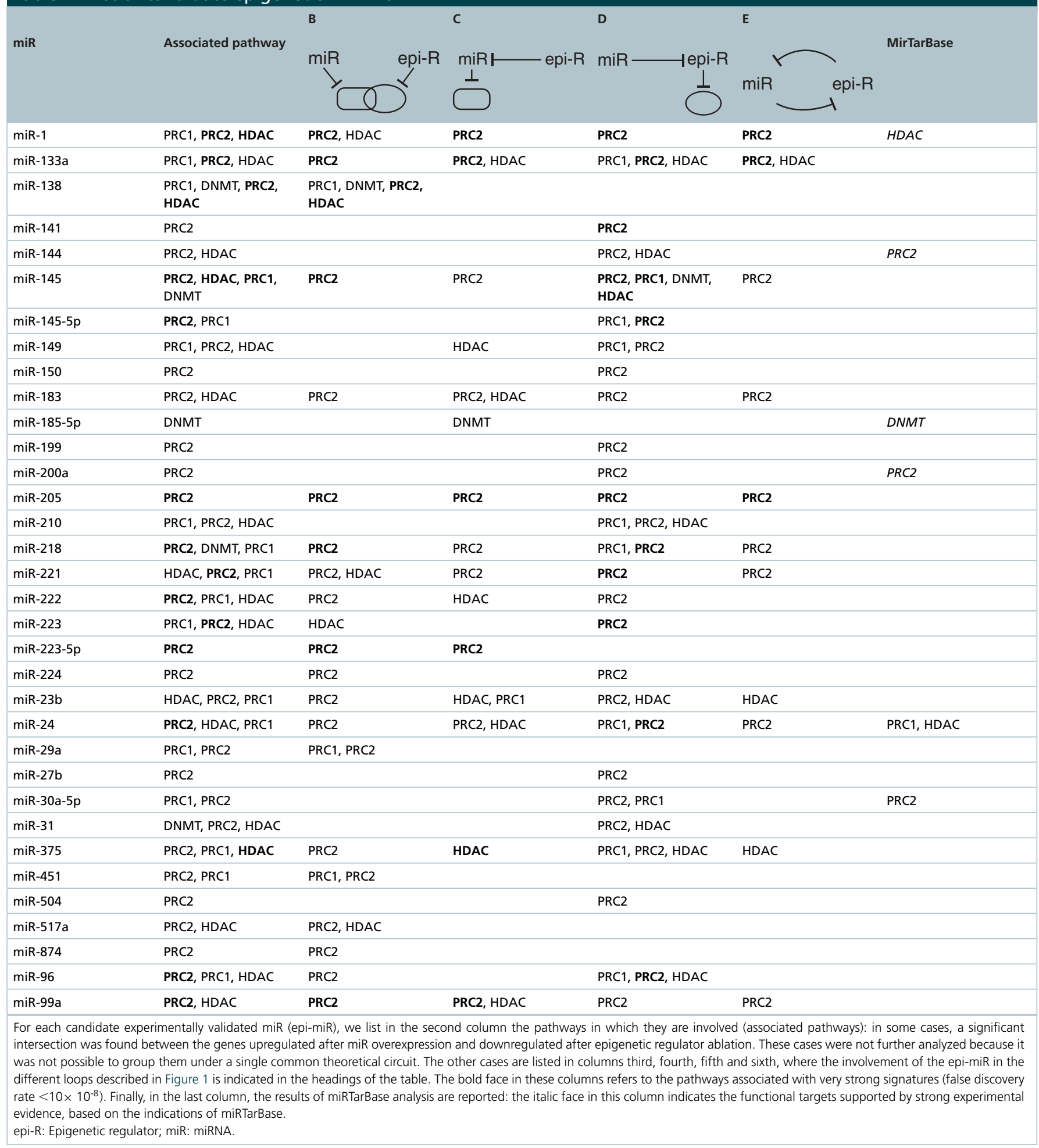

We observed that transfection experiments for our candidate epi-miRs are characterized by a large number of regulated genes (Figure 3C-D) with a slight tendency toward upregulated versus downregulated genes (Figure 3C \& Supplementary Figure 6A), a signature compatible with a widespread action of epi-Rs. As shown by the box-plot analysis, the total number of differentially expressed genes resulted significantly higher for the experiments where the miR turned out to be a candidate epi-miR compared with experiments where the miR was not identified as 
a candidate epi-miR (Figure 3D). The categorical scatter plot (Figure 4A) shows the amount of the differentially expressed genes in the different transfection experiments for each candidate epi-miR. Histogram in Figure 4B shows that the difference between the distribution of the average of the total number of differentially expressed genes for each transfection experiment and each analyzed miR is significantly higher in miRs acting as epi-miRs as assessed by Mann-Whitney U two-tailed test. Moreover, a TargetScan analysis of predicted targets of the candidate epi-miRs did not show a significant difference in number compared with the predicted target genes of the other miRs (Supplementary Figure 6B).

We describe below a few instances of our results, which we chose both for the relevance of the pathways in which the candidate epi-miRs are involved and because they represent paradigmatic examples of the different combination of entries that we found in our analysis.

\section{Examples of candidate epi-miRs identified with our pipeline miR-31}

In the panel of the analyzed transfection experiments, miR-31, a typical miR deregulated in cancer, was present in a single transfection experiment in the human highly metastatic prostate cancer cell line, PC3. Looking at the intersections with our gene sets, four entries were observed (FDR $<0.001$, see Supplementary File 7). All of them were for the set of genes upregulated after transfection (NUYTTEN_EZH2_TARGETS_UP, SENESE_HDAC1 _TARGETS_UP, SEN ESE_HDAC3_TARGETS_UP and GSE27434WT_VS_DNMT1_KO_TREG_DN). Three sets contained genes upregulated after knockout of EZH2, HDAC1 and HDAC3, suggesting a direct inhibition of these three genes by miR-31 or more generally on the corresponding epigenetic machinery as shown in the third column of Table 1.

miR-205

As for miR-31, also miR-205 was not previously known as an epi-miR. For miR-205, three experiments on three prostate cancer cell lines (PC3, DU145 and C4-2) were present in our panel. A larger number (seven) of significant intersections with our gene sets both for the upregulated and downregulated genes was observed with very low FDR (Supplementary File 7). Interestingly, for one of the EZH2-related files, the FDRs are really low (down to $10 \times 10^{-47}$ ) and genes show consistent signatures in the upregulated and downregulated sets. More precisely, the upregulated genes are the same found upregulated after knockout of EZH2 (NUYTTEN_EZH2_TARGETS_UP, FDR $=10 \times 10^{-22}$ ) and downregulated those which are downregulated after knockout of EZH2 (NUYTTEN_EZH2_TARGETS_DN, FDR $=10 \times 10^{-47}$ ).

\section{$\operatorname{miR}-138$}

Differently from the previously described miRs, miR-138 is a well-known epi-miR [7], in this case only one transfection experiment in the A498 kidney carcinoma cell line was present in the considered datasets. Eight intersections (FDR <0.001) were observed and all of them in the set of genes downregulated after transfection. Noteworthy, in all these cases, the gene sets are incoherent with respect to the expected epi-miR interaction. For instance, we found among the downregulated genes a large number $\left(F D R=10 \times 10^{-19}\right)$ of genes upregulated after knockout of EZH2. This means that these genes are simultaneously targets of miR-138 and EZH2. We report as an example the intersection corresponding to the file NUYTTEN_EZH2_TARGETS_UP mentioned above. This intersection is composed by 95 genes which are listed in the Supplementary File $7\left(\mathrm{FDR}=10 \times 10^{-19}\right)$. Performing a Gene Ontology analysis (DAVID tool online https://david.ncifcrf.gov/home.jsp) [44,45], entries related to immune response with a low Bonferroni corrected p-value of $10 \times 10^{-8}$ were observed (Figure 5A), underlining that a large portion of these genes is activated only following a perturbation of the immune system and should be otherwise kept silenced.

miR-145

The well-known epi-miR, miR-145, is the miR with the largest number of available transfection experiments, in some cases on the same cell lines. miR-145 is simultaneously involved in several different epigenetic pathways (see Table 1). In particular, we found strong evidence of a direct involvement in the regulation of the two Polycomb pathways, PRC1 and PRC2, and of the DNMT machinery. Most relevantly, the same pathway is regulated in several different cell lines, for instance, PRC2 is downregulated not only in LNCap but also in PC3 and DU145 prostate cancer cell lines. Interestingly, a direct inspection shows that the effect of the downregulation of PRC2 is 
(A)

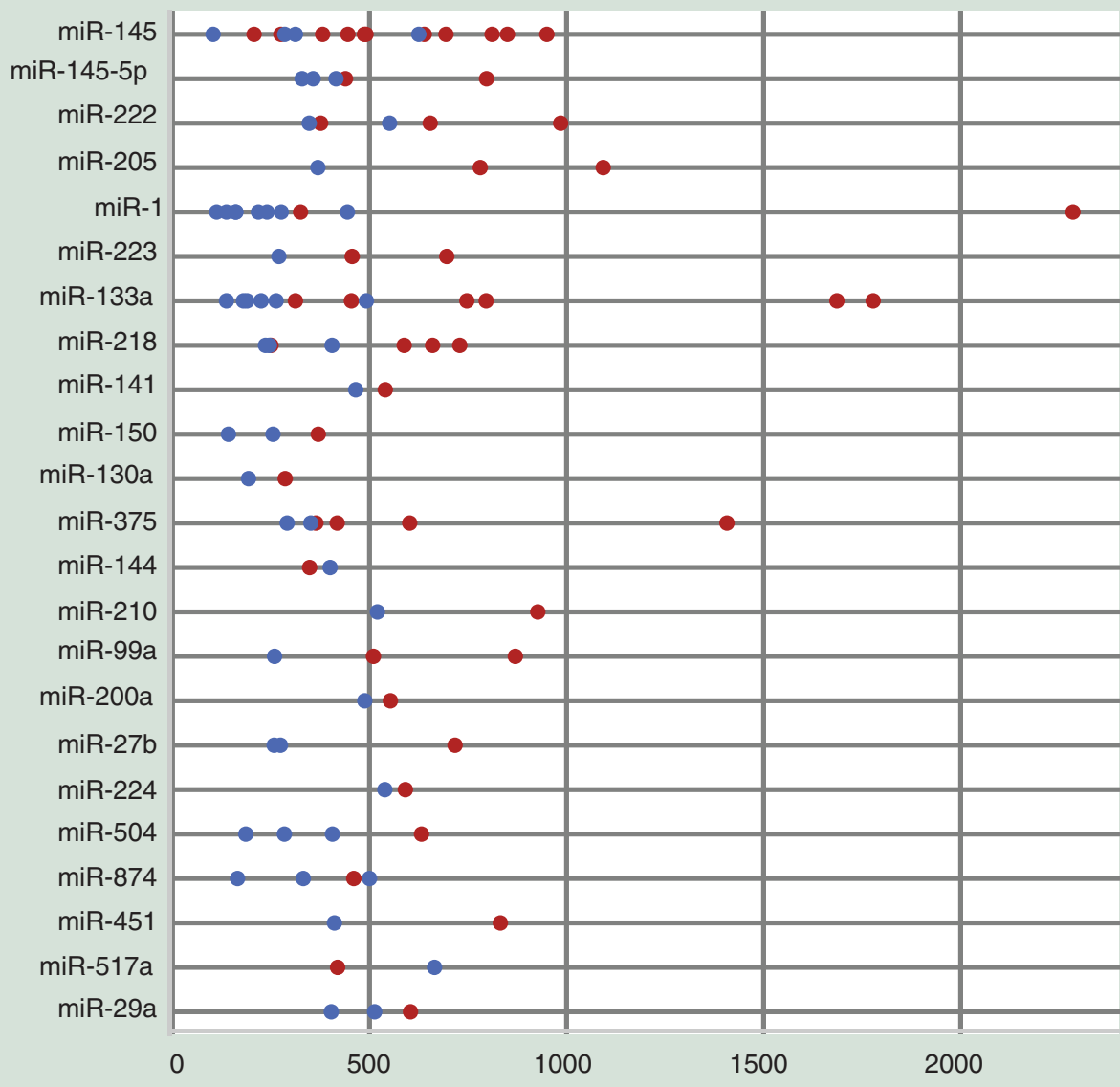

Total number of differentially expressed genes

(B)

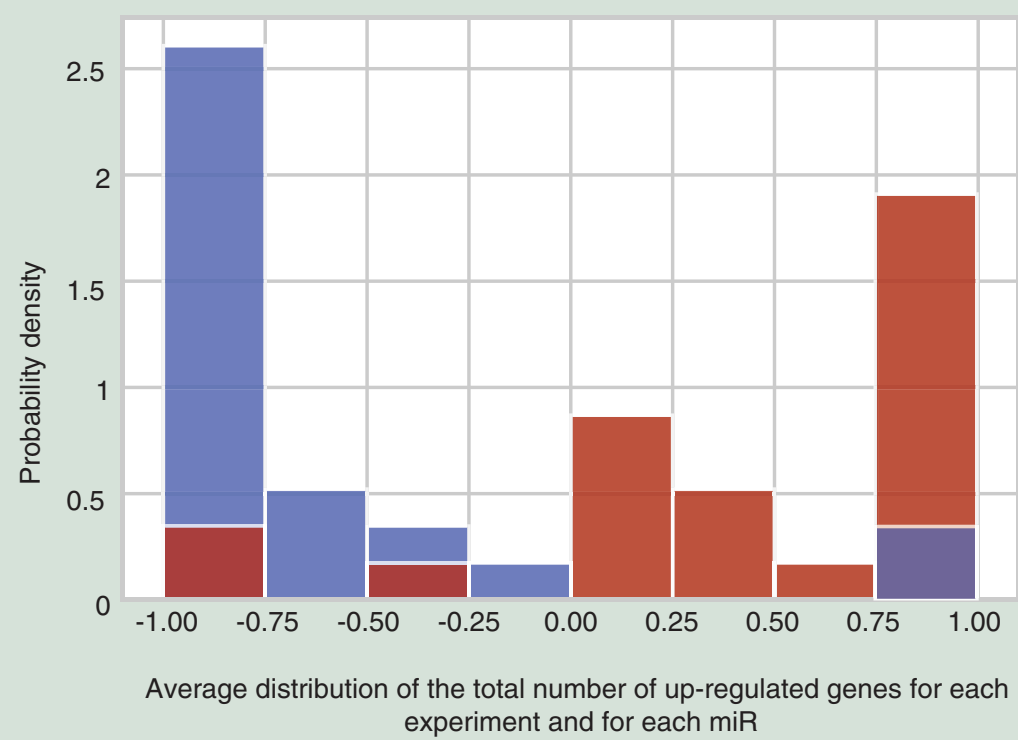

Figure 4. Single candidate experimentally validated miRNA experiments show a larger number of differentially expressed genes. (A) Categorical scatter plot representing the total number of differentially expressed genes in all the considered experiments for each identified experimentally validated miR (epi-miR). Red color indicates experiments in which the miR was considered an epi-miR (FDR threshold 0.001), while blue color indicates experiments where the miR was not considered an epi-miR (FDR $>0.001)$. (B) Histogram showing the distribution of the average of the total number of differentially expressed genes for each transfection experiments and each analyzed miR. Red: experiments where the miR was considered an epi-miR. Blue: all the experiments in which the miR was not considered an epi-miR. Mann-Whitney $U$ two-tailed test was used to assess the significance of the difference for the two distributions ( $p<0.0001$ ). miR: miRNA. 
(A)

miR-138 downregulated genes

GO:0051607_defense response to virus GO:0060337_type 1 interferon signaling pathway GO:0045071_negative regulation of viral genome replication UP_KEYWORDS_Antiviral defense GO:0006954_inflammatory response GO:0005615_extracellular space GO:0006955_immune response GO:0009615_response to virus KEGG_PATHWAY_hsa04668:TNF signaling pathway GO:0005576_extracellular region

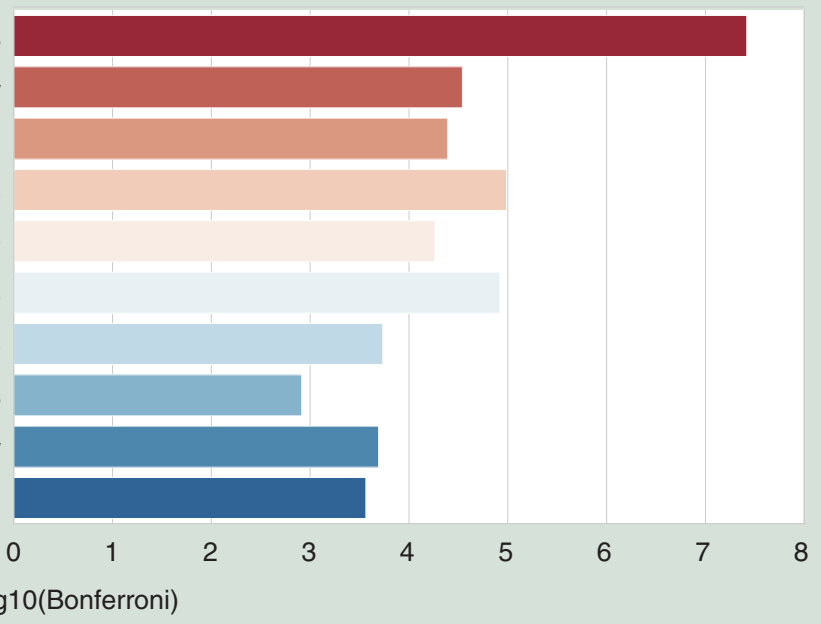

(B)

miR-1 upregulated genes

GO:0005615_extracellular space GO:0060337_type I interferon signaling pathway

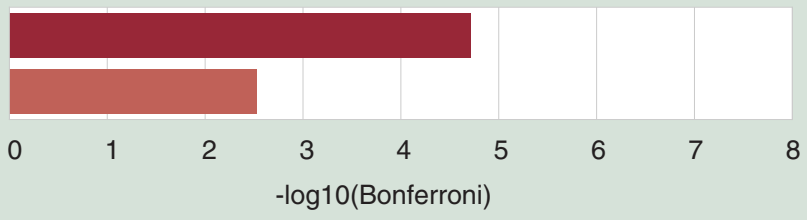

(C)

miR-1 downregulated genes

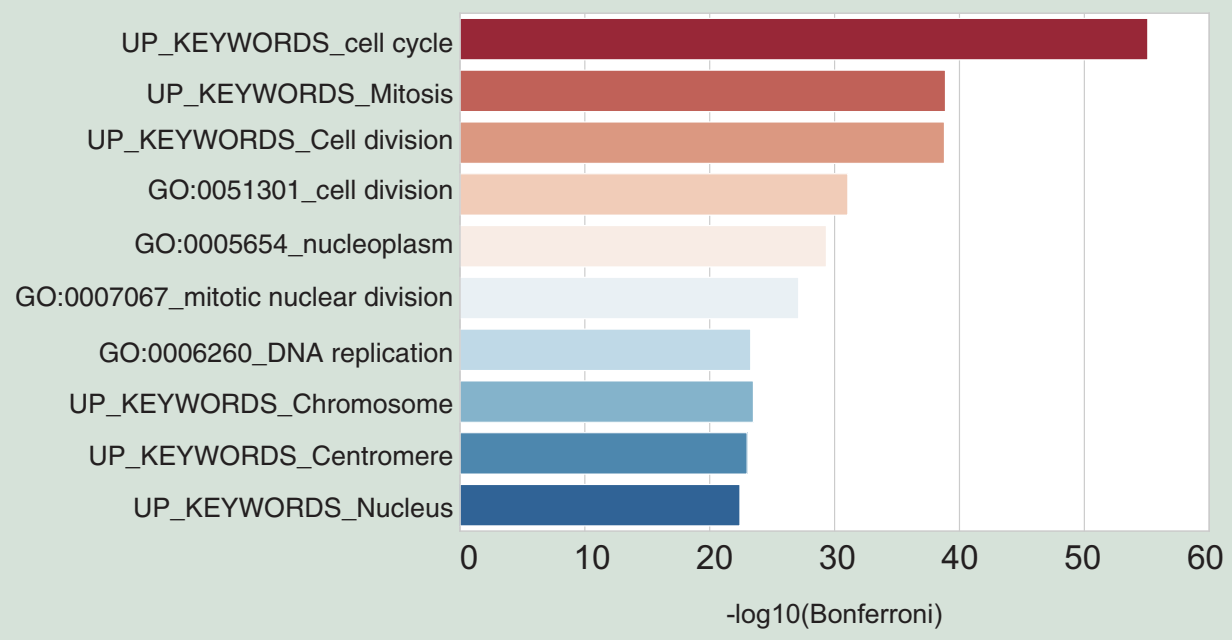

Figure 5. Gene ontology analysis for miR-138 and miR-1. (A-C) Bar plots of the best entries for Database for Annotation, Visualization and Integrated Discovery gene ontology analysis performed for the genes of the best intersections with gene set enrichment analysis sets obtained for (A) miR-138 and (B-C) miR-1. For each entry, the log10 of the Bonferroni corrected p-value is shown (cutoff on the Bonferroni corrected $\mathrm{p}=0.001)$. 
very similar in the three cell lines. In other words, there is a large overlap among the genes that are upregulated in the three experiments (notwithstanding the difference in cell lines) and these upregulated genes are exactly those which are targeted by PRC2 (data not shown).

miR-1

A very interesting pattern was found in the case of the well-known epi-miR, miR-1 [7], which appeared in ten entries of our panel of transfection experiments, but only for two of them, corresponding to the PC3 (prostate cancer) and EBC-1 (lung cancer) cell lines, the intersection had a FDR $<0.001$. In particular, in lung cancer EBC-1 cells, there was a strong indication supporting a direct downregulation of PRC2. What is relevant is that this signature appears in a consistent way both in the set of the upregulated and downregulated genes (see data in Supplementary File 7). We found a large overlap $\left(\mathrm{FDR}=10 \times 10^{-73}\right)$ between the set of genes downregulated after transfection of the miR and the genes downregulated after knockout of EZH2 and, simultaneously, a large overlap (FDR = 10 $\left.\times 10^{-16}\right)$ of the genes upregulated after transfection with those upregulated after knockout of EZH2. The set of common upregulated genes shows a strong overlap with the same GO categories mentioned above on immune response (Figure $5 \mathrm{~B}$ ), while a $\mathrm{GO}$ analysis of the set of downregulated genes shows that most of these genes are associated to cell cycle (Figure 5C).

GO categories for miR-31, miR-145 and miR-205 are shown in Supplementary Figure 8.

\section{miR-214 modulates multiple genes in melanoma cells}

The procedure we propose can be used given a miR of interest, to identify its candidate epigenetic interactors. Due to the relevance of miR-214 in tumor progression, we are continuously investigating unknown mechanisms through which miR-214 could coordinate tumor metastatization. In the attempt to identify new genes directly or indirectly modulated by miR-214, MA-2 [19] aggressive melanoma cells were transiently transfected with a precursor for miR-214 (pre-miR-214) or negative controls (precontrol). RNA was extracted $72 \mathrm{~h}$ following transient transfection and miR-214 expression levels verified by real-time PCR (data not shown). RNA was used to perform whole human genome gene expression analysis (GSE124965). A total of 493 differentially expressed genes (342 upregulated; 88 downregulated) were found, considering a 14.2\% FDR. Surprisingly, 342/493 of the differentially expressed genes resulted to be upregulated, suggesting a possible indirect regulation of these genes by miR-214 via a potential crosstalk with an epi-R. Similar observation came from the analysis of another miR-214 dataset (GSE35948) [39]. The first hypothesis we made was that miR-214 could influence the expression of this huge amount of genes via an epigenetic mechanism. For this reason, the list of the differentially expressed genes was subjected to the analysis described above to identify candidate epigenetic interactors of miR-214. The lists of the differentially expressed genes used for the analysis is available in Supplementary File 9. The heatmaps for both datasets are shown in Figure $6 \mathrm{~A}$ and $\mathrm{B}$, where it is qualitatively appreciable the increased number of upregulated genes compared with downregulated ones following miR-214 overexpression.

Eight GSEA sets were identified, across up- and downregulated gene sets using both our (GSE124965) and GSE35948 [39] datasets (Supplementary File 10), showing evidence of a direct regulation of the miR on the considered epigenetic pathways. Five of them correspond to the PRC2 pathway and two correspond to HDACs. In particular, the PRC2 sets are related to the silencing of the enhancer of zeste 2 polycomb-repressive complex 2 subunit (EZH2) and to the silencing of the EZH2 interactor NIPP1 (Supplementary File 10), suggesting EZH2 as the candidate gene directly targeted by miR-214 and responsible for the vast upregulation observed in the experimental dataset. GSEA analysis of the genes belonging to the best PRC2 intersection, described above, shows a significant enrichment with sets related to epithelial-to-mesenchymal transition and TGFb pathway in agreement with the prometastatic role of miR-214 (Figure 7).

\section{miR-214 downregulates EZH2}

In order to prove that miR-214 targets EZH2 gene in a melanoma cell context, miR-214 was overexpressed in MA-2 melanoma cells and EZH2 protein expression levels analyzed. As shown in Figure 8A, miR-214 overexpression leads to a $60 \%$ reduction of EZH2 protein levels. To verify if EZH2 silencing could phenocopy miR-214 overexpression effects on the activation of miR-214-dependent downstream pathway, ITGA5 and ALCAM, and miR-148b expression levels were analyzed. As shown in Figure 8B and C, EZH2 silencing (60\%) was able to induce an upregulation of ALCAM (32\%) and ITGA5 (60\%) expression, thus phenocopying the effects observed following miR-214 overexpression [20]. Interestingly, ALCAM and ITGA5 were not consistently modulated at the mRNA 


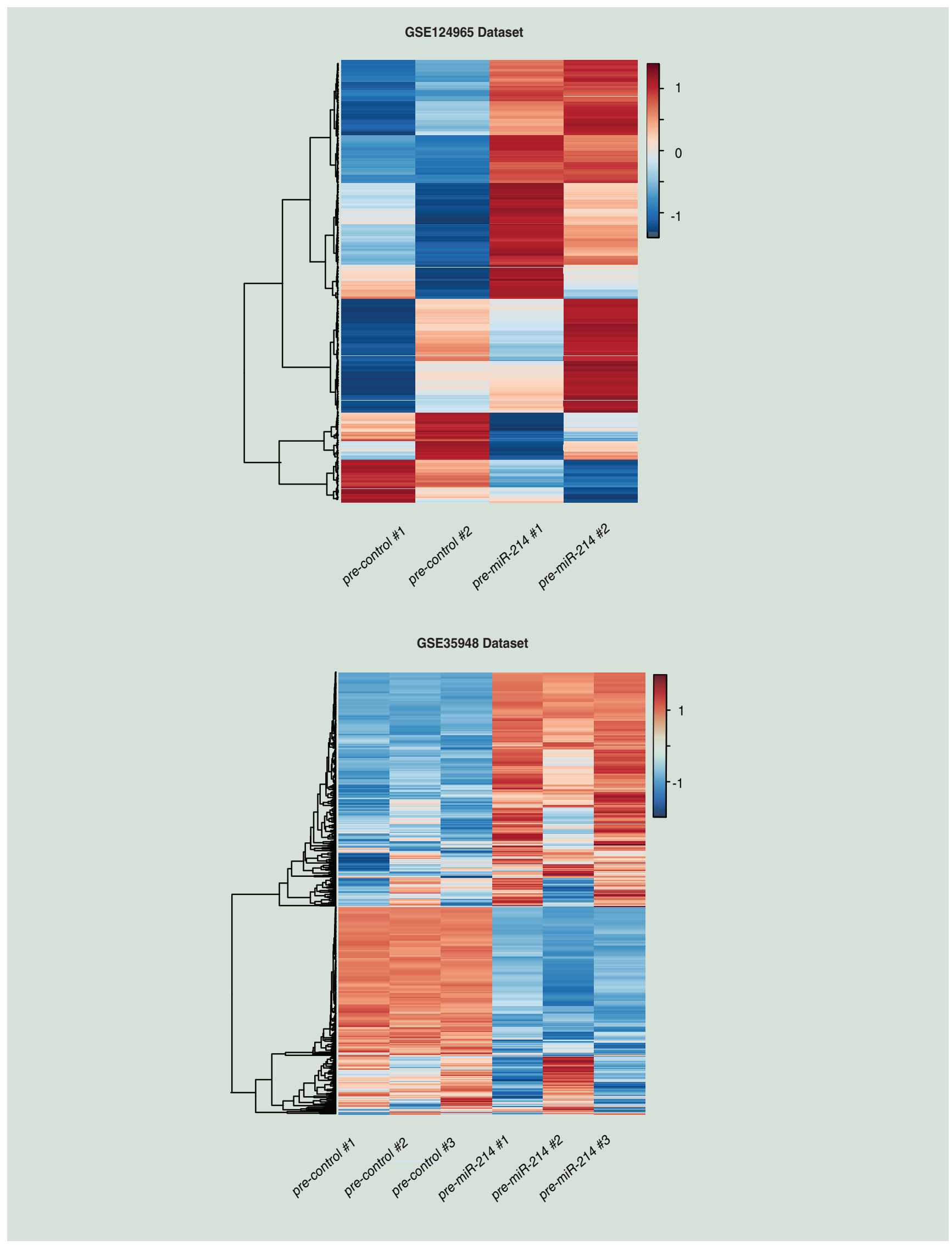

Figure 6. Heatmaps of the expression levels of miR-214-regulated genes. (A) Heatmap representing the results of the microarray analysis performed on MA-2 melanoma cells $72 \mathrm{~h}$ following miR-214 transient overexpression (GSE124965). (B) Heatmap representing the results of the microarray analysis of GSE35948 dataset. 


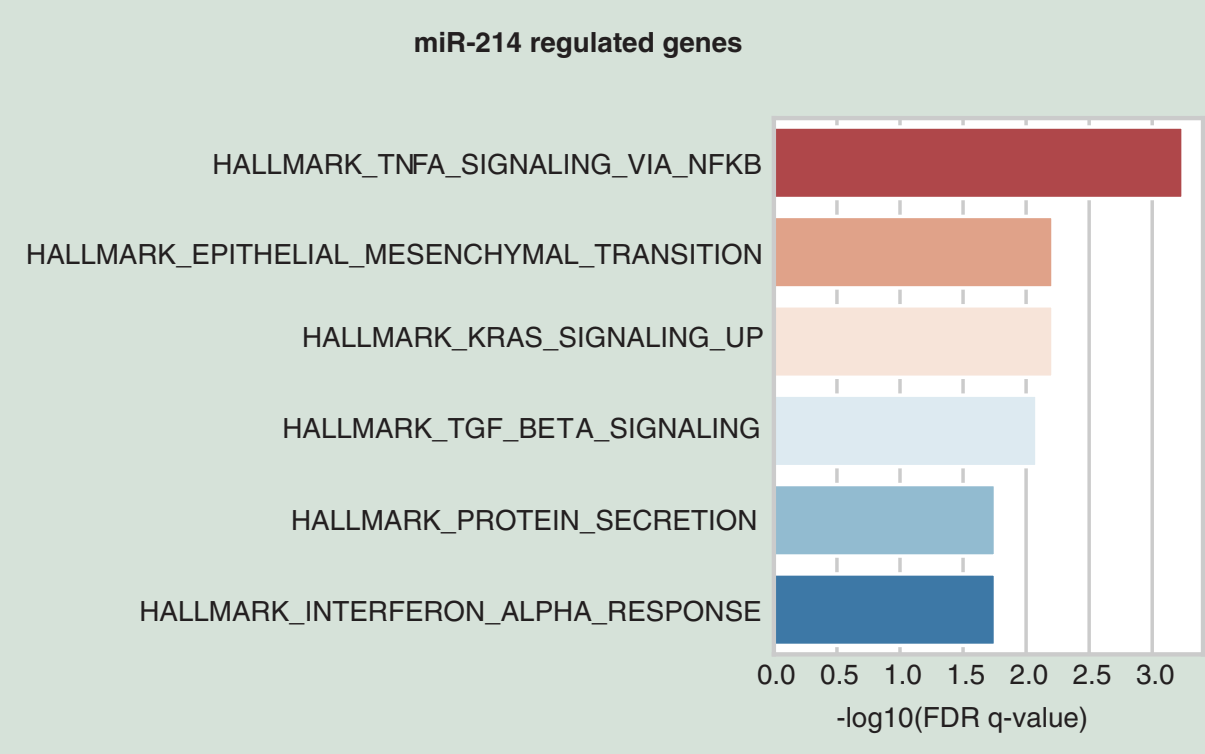

Figure 7. Functional enrichment for the best miR-214 intersection with gene set enrichment analysis sets. Best entries for enrichment analysis performed on gene set enrichment analysis hallmark collection for the best intersection of miR-214 regulated genes with gene set enrichment analysis sets. For each entry, the log10 of the FDA q-values is shown.

FDA: False discovery rate.

level. In order to asses if EZH2 could be partially involved in miR-214-mediated miR-148b dowmmodulation, we tested miR-148b expression levels following EZH2 silencing and as shown in Figure 8D. EZH2 silencing was able to decrease miR-148b expression, suggesting a partial contribution of EZH2 in miR-214-mediated control of miR-148b. All these data suggest that miR-214 could potentially act, at least partially, through an epigenetic mechanism together with the classical mechanism of action in controlling metastatization.

\section{Discussion}

We selected a panel of gene sets which can be considered bona-fide targets of the above-discussed main epigenetic pathways and intersected them with the set of genes, respectively, upregulated or downregulated in a series of gene expression datasets coming from publicly available transfection experiments of various miRs. Intuitively, a large intersection could be considered as a signature of an epigenetic amplification. The p-value of the intersection was assessed using the hypergeometric distribution corrected for multiple testing. Our analysis shows that the interaction between miRs and epi-Rs is widespread. Epi-miRs are typically involved in differentiation processes and are likely to play an important role in cancer, where accumulating evidence shows that epigenetic alterations exert pivotal roles in tumor progression. When transfection experiments in more than one cell line were available, we found that the epigenetic role of miRs is strongly tissue dependent, in fact, the same miR can strongly interact with an epi-R in a particular tissue or cancer cell line and may have no effect at all in another cell line.

An interesting feature of most of the known epi-miRs is that they are typically involved in complex double inhibitory feedback loops with their epigenetic targets. It has been shown that this circuit is perfectly suited for tissue differentiation and more generally to choose among different cell fates and keep memory of this choice [12]. The nature of the two partners ( $\mathrm{miR}$ and epi-R) of the feedback loop ensures an optimal resistance to stochastic fluctuations and avoids erroneous switching of the loop [12]. Several of the identified epi-miRs follow this same pattern, but due to the nature of the data used in our analysis we cannot close the circuits for all the identified candidate epi-miR except for the ones presented in Table 1, column sixth. We discuss here a few examples of epi-miRs which are presented in the Results section.

MiR-31 is an important regulator of tumorigenesis where it can function either as an oncomiR or as a tumorsuppressive miR. This miR was not previously known as an epi-miR and there are no entries in the miRTarBase. miR-31 has been recently shown to be a transcriptional target of EZH2 [46,47]. Together with our findings, this 
(A)

MA-2

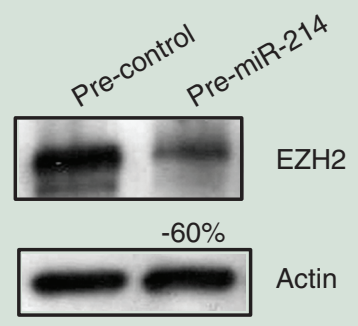

(B)

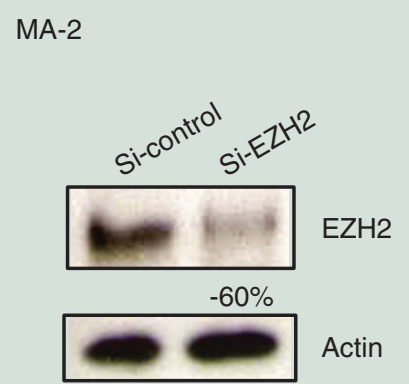

(C)
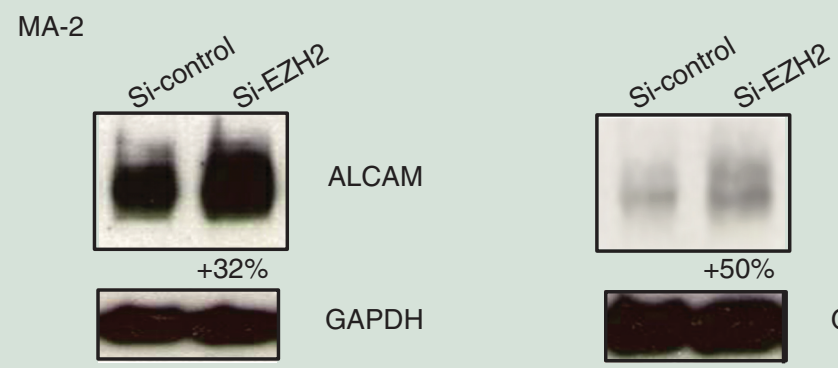

ITGA5

GAPDH
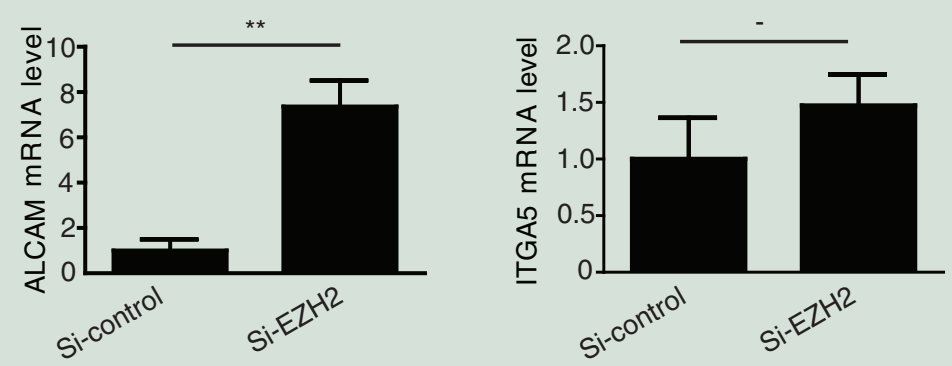

(D)

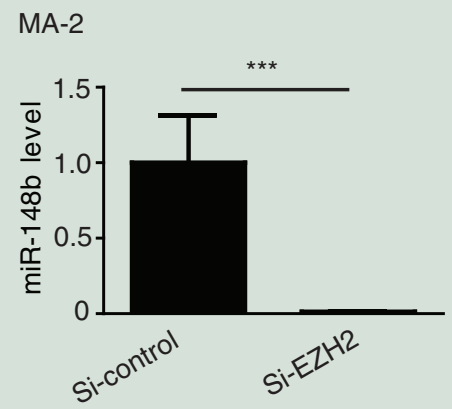

Figure 8. Histone methyltransferase enhancer of zeste homolog 2 is a target of miR-214. (A \& B) EZH2 protein levels were assessed by WB in MA-2 cells $72 \mathrm{~h}$ after transfection with miR-214 precursors or their negative controls (pre-214 vs control) or after EZH2 transient downmodulation (siEZH2 vs si-control). (C) ALCAM and ITGA5 protein and mRNA levels were assessed by Western blot and quantitative reverse transcription-PCR in MA-2 cells $72 \mathrm{~h}$ after EZH2 transient downmodulation (siEZH2 vs si-control). (D) miR-148b expression levels were assessed by quantitative reverse transcription-PCR in MA-2 cells $72 \mathrm{~h}$ after EZH2 transient downmodulation (siEZH2 vs si-control). Protein modulations were calculated relative to controls, normalized on Actin (B \& C) or GAPDH (D) loading controls and expressed as percentages; results in (C) and (D) are shown as fold changes (mean \pm standard error of the mean) relative to controls, normalized on GAPDH mRNA or U6 snRNA levels. Two experiments with independent protein and RNA preparations were performed and representative ones are shown.

EZH2: Histone methyltransferase enhancer of zeste homolog 2.

observation closes a perfect toggle switch (see Figure 1E) which, as mentioned above, is a typical signature of epi-miRs. In agreement with this switch-like behavior, it was shown that in colorectal cancer cell lines [46], there is a systematic inverse association between EZH2 and miR-31 expression levels.

Remarkably enough, it has been recently shown that a similar feedback loop is also present with the other epigenetic pathways. Indeed, it was shown that HDAC inhibitors enhance the expression level of miR-31 [48]. Moreover, also the association between the HDAC and the PRC2 pathways that we observed in our data is 
confirmed in the literature, in fact, it has been shown recently that in some classes of tumors the inhibition of miR-31 requires the joint action of EZH2 and HDAC [49].

As for the previous miR, also miR-205 may act either as a tumor suppressor through inhibiting proliferation and invasion, or as an oncogene through facilitating tumor initiation and proliferation, depending on the specific tumor context and target genes. miR-205 belongs to the miR-200 family and is involved in the regulation of EMT. As for miR-31 also miR-205 was not previously reported as an epi-miR. Also in this case, there is evidence of a double inhibitory feedback loop with EZH2 [50].

Differently from the previously described miRs, miR-138 is a well-known epi-miR, it is involved in a doublenegative feedback loop both with SIRT1 [51], which is a component of the HDAC pathway and with EZH2 [52,53]. These circuits are prototypical examples of epigenetic feedback loops [12]. What is interesting is that in all these cases, the gene sets are incoherent with respect to the expected epi-miR interaction. This means that these genes are simultaneously targets of miR-138 and EZH2. The type of circuit that we found is depicted in Figure 1B. This circuit is very interesting because it ensures that the target genes are always silenced, independently from the orientation of the regulatory switch. What is more important, due to the incoherent nature of the circuit, their expression levels are not only low but are stable against fluctuations. These target genes are likely to be important regulator themselves, which in physiological conditions must be kept under control while are disregulated during cancer progression.

miR-145 is a known tumor suppressor that impacts on tumor cell growth and invasiveness, plays important roles in the differentiation of stem cells and vascular smooth muscle cells and regulates the expression of core stemnessassociated factors, such as OCT4, SOX2 and KLF4 [54]. It is interesting to notice that also for this miR, there are strong indications suggesting the presence of a double inhibitory feedback loop with DNMT [55]. This agrees with independent observations: for instance, it was shown to be suppressed by hypermethylation in tumors [56] and it is downregulated in lung adenocarcinoma tissues in association with increased DNA methylation [57]. The DNMT pathway is indeed one of the pathways that we found as direct targets of miR-145 in our analysis (see Table 1). Finally, a very interesting pattern is found in the case of the well-known miR-7 [7], for which GO categories show an enrichment in immune response and cell cycle inhibition categories and our findings suggest that it exerts these functions by targeting the PRC2 pathway.

Our pipeline can be used to identify putative epigenetic interactors of any given miR. The prometastatic miR-214 has been used as an example of the potentiality of our methods. Based both on a dataset from our transfection experiments and from a publicly available dataset, we identified EZH2 as an epigenetic interactor of miR-214. We demonstrated that miR-214 regulates EZH2 in melanoma cells and EZH2 is, in turn, at least partially responsible of the downstream pathway activated by miR-214 involving ALCAM, ITGA5 and miR-148b. These results were in agreement with previous data regarding miR-214 regulation of EZH2 during muscle differentiation [58]. Moreover, the GSEA analysis performed for the best intersection of miR-214-regulated genes with the selected GSEA sets used in our analysis shows an enrichment in EMT and TGF- $\beta$-related genes, perfectly in agreement with the prometastatic role of miR-214 during tumor progression. The identification of miR-214 as an epi-miR in a tumor cell context highlights new layers of regulation of this $\mathrm{miR}$ in the control of tumor progression.

\section{Conclusion}

Some of our candidate epi-miRs agree with already published findings, and represent a positive test of our procedure, but some of them are new and represent one of the main results of this paper. Altogether our findings point to a much stronger role of epi-miR in the regulatory network of higher eukaryotes and more generally to a strong interplay between the post-transcriptional and the epigenetic layers of regulation in shaping the differentiation process of complex tissues.

\section{Future perspective}

The alteration of epigenetic mechanisms can cause several serious pathologies, including cancers, cardiovascular, metabolic, neurodegenerative disorders as well as inherited syndromes. Recently, growing evidence demonstrates that miRs influence epigenetic mechanisms, via regulation of epigenetic factors, and at the same time, many miRs are under a strict epigenetic control. Given the importance and complexity of this interplay, the epigenetic mechanisms can be only understood considering the regulatory circuitries composed of miRs and epigenetic regulators. Our work contributes to better elucidate and to identify new player involved in these complex regulatory circuits. Although the presented pipeline was applied on gene sets from tumor origin, it could be applied to different pathologies 
beside cancers. Notably, our approach could be used in the future to go more in details in this regulations to verify how much these circuitries are similar among cells of the same tissue of origin or cells derived from the same kind of tumors as well as to unravel the pathological implications of new epimiR. Potentially, the level of resolution could also be increased in order to discriminate among the different members of the epigenetic regulator family involved in the circuits. Moreover, an enhanced resolution will also help to better elucidate and dissect the molecular mechanism. Due to the relevance of these regulatory circuitries in the future, many more studies and new methodologies will be developed to shed light on this new level of regulation.

\section{Summary points}

- miRNAs (miRs) are small noncoding RNAs able to post-transcriptionally regulate gene expression.

- miRs can cross-talk with other regulatory genes such as the epigenetic factors, amplifying their regulatory potential.

- miRs-regulating epigenetic factors are denoted as epi-miRNAs (epi-miRs).

- Starting from pre-existing miR transfection experiments, we identified 34 candidate epi-miRs: 19 of them are known as epi-miRs, while 15 are new.

- We found that the same miR may act as an epi-miR in one particular cell line and show no epigenetic effect in the other cell lines.

- Using an in-house generated gene expression dataset, we experimentally proved that, a component of the polycomb-repressive complex 2, histone methyltransferase enhancer of zeste homolog 2 (EZH2), is an interactor of miR-214, a well-known prometastatic miR in melanoma and breast cancer.

\section{Author contributions}

M Caselle and F Orso contributed to the conception, design and supervision. E Reale, M Osella, M Caselle, F Orso, L Martignetti and L Cantini contributed to the development and methodology. E Reale, M Caselle, F Orso, F Virga, C De Pittà, D Taverna, L Martignetti and L Cantini contributed to the data acquisition, analysis and interpretation. M Caselle, E Reale, M Osella and F Orso wrote the manuscript.

\section{Acknowledgments}

The authors are grateful to Lei Xu and Richard Hynes for the MA-2 cell line.

Financial \& competing interests disclosure

M Caselle received the funds from Departments of Excellence 2018-2022 Italian Ministry of University and Research (MIUR; L.232/2016). F Orso funded by FIRB giovani 2008 project (RBFR08F2FS-002 FO). D Taverna received the funds Compagnia di San Paolo, Torino, 2008.1054, AIRC 2010, 2013, 2017 (IG2010-10104DT; IG2013-14201DT; IG2017-20258DT), Fondazione Cassa di Risparmio Torino CRT (2014.1085DT); Progetto di Ricerca di Ateneo 2017/San Paolo Torino CST0165134. The authors have no other relevant affiliations or financial involvement with any organization or entity with a financial interest in or financial conflict with the subject matter or materials discussed in the manuscript apart from those disclosed.

No writing assistance was utilized in the production of this manuscript.

\section{Open Access}

This work is licensed under the Attribution-NonCommercial-NoDerivatives 4.0 Unported License. To view a copy of this license, visit http://creativecommons.org/licenses/by-nc-nd/4.0/

\section{References}

Papers of special note have been highlighted as: • of interest; $\bullet \bullet$ of considerable interest

1. Friedman RC, Farh KK, Burge CB, Bartel DP. Most mammalian mRNAs are conserved targets of microRNAs. Genome Res. 19(1), 92-105 (2009).

2. Lewis BP, Burge CB, Bartel DP. Conserved seed pairing, often flanked by adenosines, indicates that thousands of human genes are microRNA targets. Cell 120(1), 15-20 (2005).

3. Ambros V. The functions of animal microRNAs. Nature 431(7006), 350-355 (2004).

4. Bartel DP. MicroRNAs: genomics, biogenesis, mechanism, and function. Cell 116(2), 281-297 (2004).

5. Alvarez-Garcia I, Miska EA. MicroRNA functions in animal development and human disease. Development 132(21), 4653-4662 (2005).

6. Esquela-Kerscher A, Slack FJ. Oncomirs - microRNAs with a role in cancer. Nat. Rev. Cancer 6(4), 259-269 (2006).

7. Gruber AJ, Zavolan M. Modulation of epigenetic regulators and cell fate decisions by miRNAs. Epigenomics 5(6), 671-683 (2013). 
•• Comprehensive review on experimentally tested epi-miRNAs and on their involvement in different pathologies

8. Iorio MV, Piovan C, Croce CM. Interplay between microRNAs and the epigenetic machinery: an intricate network. Biochim. Biophys. Acta 1799(10-12), 694-701 (2010).

9. Kunej T, Godnic I, Ferdin J, Horvat S, Dovc P, Calin GA. Epigenetic regulation of microRNAs in cancer: an integrated review of literature. Mutat. Res. 717(1-2), 77-84 (2011).

10. Sato F, Tsuchiya S, Meltzer SJ, Shimizu K. MicroRNAs and epigenetics. FEBS J. 278(10), 1598-1609 (2011).

11. Wang Z, Yao H, Lin S et al. Transcriptional and epigenetic regulation of human microRNAs. Cancer Lett. 331(1), 1-10 (2013).

12. Osella M, Riba A, Testori A, Cora D, Caselle M. Interplay of microRNA and epigenetic regulation in the human regulatory network. Front. Genet. 5, 345 (2014).

- Paper on the modelization, using stochastic equations, of the feedback loop between epi-miRNAs and epigenetic regulators

13. Di Croce L, Helin K. Transcriptional regulation by Polycomb group proteins. Nat. Struct. Mol. Biol. 20(10), 1147-1155 (2013).

14. Nuytten M, Beke L, Van Eynde A et al. The transcriptional repressor NIPP1 is an essential player in EZH2-mediated gene silencing. Oncogene 27(10), 1449-1460 (2008).

15. Weber M, Hellmann I, Stadler MB et al. Distribution, silencing potential and evolutionary impact of promoter DNA methylation in the human genome. Nat. Genet. 39(4), 457-466 (2007).

16. Haberland M, Montgomery RL, Olson EN. The many roles of histone deacetylases in development and physiology: implications for disease and therapy. Nat. Rev. Genet. 10(1), 32-42 (2009).

17. Habibian J, Ferguson BS. The crosstalk between acetylation and phosphorylation: emerging new roles for HDAC inhibitors in the heart. Int. J. Mol. Sci. 20(1), pii: E102 (2018).

18. Bommi PV, Dimri M, Sahasrabuddhe AA, Khandekar J, Dimri GP. The polycomb group protein BMI1 is a transcriptional target of HDAC inhibitors. Cell Cycle 9(13), 2663-2673 (2010).

19. Penna E, Orso F, Cimino D et al. MicroRNA-214 contributes to melanoma tumour progression through suppression of TFAP2C. EMBO J. 30(10), 1990-2007 (2011).

- Paper highlighting the relevance of miR-214 in the progression of melanoma.

20. Penna E, Orso F, Cimino D et al. miR-214 coordinates melanoma progression by upregulating ALCAM through TFAP2 and miR-148b downmodulation. Cancer Res. 73(13), 4098-4111 (2013).

- Paper unraveling the complex regulatory network involving both coding and noncoding genes controlled by miR-214 during melanoma progression.

21. Mahmoud F, Shields B, Makhoul I, Hutchins LF, Shalin SC, Tackett AJ. Role of EZH2 histone methyltrasferase in melanoma progression and metastasis. Cancer Biol. Ther. 17(6), 579-591 (2016).

22. Liberzon A, Subramanian A, Pinchback R, Thorvaldsdottir H, Tamayo P, Mesirov JP. Molecular signatures database (MSigDB) 3.0. Bioinformatics 27(12), 1739-1740 (2011).

23. Subramanian A, Tamayo P, Mootha VK et al. Gene set enrichment analysis: a knowledge-based approach for interpreting genome-wide expression profiles. Proc. Natl Acad. Sci. USA 102(43), 15545-15550 (2005).

- Standard reference on the gene set enrichment analysis and on the Molecular Signature database used in this paper.

24. Chiyomaru T, Yamamura S, Fukuhara $S$ et al. Genistein inhibits prostate cancer cell growth by targeting miR-34a and oncogenic HOTAIR. PLOS ONE 8(8), e70372 (2013).

25. Fuse M, Kojima S, Enokida $\mathrm{H}$ et al. Tumor suppressive microRNAs (miR-222 and miR-31) regulate molecular pathways based on microRNA expression signature in prostate cancer. J. Hum. Genet. 57(11), 691-699 (2012).

26. Hidaka H, Seki N, Yoshino $\mathrm{H}$ et al. Tumor suppressive microRNA-1285 regulates novel molecular targets: aberrant expression and functional significance in renal cell carcinoma. Oncotarget 3(1), 44-57 (2012).

27. Kikkawa N, Kinoshita T, Nohata N et al. microRNA-504 inhibits cancer cell proliferation via targeting CDK6 in hypopharyngeal squamous cell carcinoma. Int. J. Oncol. 44(6), 2085-2092 (2014).

28. Kinoshita T, Hanazawa T, Nohata N et al. Tumor suppressive microRNA-218 inhibits cancer cell migration and invasion through targeting laminin-332 in head and neck squamous cell carcinoma. Oncotarget 3(11), 1386-1400 (2012).

29. Kinoshita T, Nohata N, Yoshino H et al. Tumor suppressive microRNA-375 regulates lactate dehydrogenase B in maxillary sinus squamous cell carcinoma. Int. J. Oncol. 40(1), 185-193 (2012).

30. Kumamoto T, Seki N, Mataki H et al. Regulation of TPD52 by antitumor microRNA-218 suppresses cancer cell migration and invasion in lung squamous cell carcinoma. Int. J. Oncol. 49(5), 1870-1880 (2016).

31. Moriya Y, Nohata N, Kinoshita T et al. Tumor suppressive microRNA-133a regulates novel molecular networks in lung squamous cell carcinoma. J. Hum. Genet. 57(1), 38-45 (2012).

32. Nohata N, Hanazawa T, Kikkawa N et al. Tumor suppressive microRNA-375 regulates oncogene AEG-1/MTDH in head and neck squamous cell carcinoma (HNSCC). J. Hum. Genet. 56(8), 595-601 (2011). 
33. Nohata N, Hanazawa T, Kikkawa N et al. Tumour suppressive microRNA-874 regulates novel cancer networks in maxillary sinus squamous cell carcinoma. Br. J. Cancer 105(6), 833-841 (2011).

34. Nohata N, Hanazawa T, Kikkawa $\mathrm{N}$ et al. Identification of novel molecular targets regulated by tumor suppressive miR-1/miR-133a in maxillary sinus squamous cell carcinoma. Int. J. Oncol. 39(5), 1099-1107 (2011).

35. Nohata $\mathrm{N}$, Sone $\mathrm{Y}$, Hanazawa $\mathrm{T}$ et al. $\mathrm{miR}-1$ as a tumor suppressive microRNA targeting TAGLN2 in head and neck squamous cell carcinoma. Oncotarget 2(1-2), 29-42 (2011).

36. Yamada Y, Koshizuka K, Hanazawa T et al. Passenger strand of miR-145-3p acts as a tumor-suppressor by targeting MYO1B in head and neck squamous cell carcinoma. Int. J. Oncol. 52(1), 166-178 (2018).

37. Yamasaki T, Seki N, Yamada Y et al. Tumor suppressive microRNA138 contributes to cell migration and invasion through its targeting of vimentin in renal cell carcinoma. Int. J. Oncol. 41(3), 805-817 (2012).

38. Yonemori K, Seki N, Kurahara $\mathrm{H}$ et al. ZFP36L2 promotes cancer cell aggressiveness and is regulated by antitumor microRNA-375 in pancreatic ductal adenocarcinoma. Cancer Sci. 108(1), 124-135 (2017).

39. Misiewicz-Krzeminska I, SarasqueteME, QuwaiderD et al. Restoration of microRNA-214 expression reduces growth of myeloma cells through positive regulation of P53 and inhibition of DNA replication. Haematologica 98(4), 640-648 (2013).

40. Durinck S, Spellman PT, Birney E, Huber W. Mapping identifiers for the integration of genomic datasets with the R/Bioconductor package biomaRt. Nat. Protoc. 4(8), 1184-1191 (2009).

41. Chou CH, Shrestha S, Yang CD et al. miRTarBase update 2018: a resource for experimentally validated microRNA-target interactions. Nucleic Acids Res. 46(D1), D296-D302 (2018).

42. Agarwal V, Bell GW, Nam JW, Bartel DP. Predicting effective microRNA target sites in mammalian mRNAs. Elife 4, (2015).

43. Cimino D, De Pitta C, Orso F et al. miR148b is a major coordinator of breast cancer progression in a relapse-associated microRNA signature by targeting ITGA5, ROCK1, PIK3CA, NRAS, and CSF1. FASEB J. 27(3), 1223-1235 (2013).

44. Huang da W, Sherman BT, Lempicki RA. Systematic and integrative analysis of large gene lists using DAVID bioinformatics resources. Nat. Protoc. 4(1), 44-57 (2009).

45. Huang da W, Sherman BT, Lempicki RA. Bioinformatics enrichment tools: paths toward the comprehensive functional analysis of large gene lists. Nucleic Acids Res. 37(1), 1-13 (2009).

46. KuriharaH, Maruyama R, Ishiguro K et al. The relationship between EZH2 expression and microRNA-31 in colorectal cancer and the role in evolution of the serrated pathway. Oncotarget 7(11), 12704-12717 (2016).

47. Zhang Q, Padi SK, Tindall DJ, Guo B. Polycomb protein EZH2 suppresses apoptosis by silencing the proapoptotic miR-31. Cell Death Dis. 5, e1486 (2014).

48. Cho JH, Dimri M, Dimri GP. MicroRNA-31 is a transcriptional target of histone deacetylase inhibitors and a regulator of cellular senescence. J. Biol. Chem. 290(16), 10555-10567 (2015).

49. Koumangoye RB, Andl T, Taubenslag KJ et al. SOX4 interacts with EZH2 and HDAC3 to suppress microRNA-31 in invasive esophageal cancer cells. Mol. Cancer 14, 24 (2015).

50. Wang S, Huang J, Lyu H et al. Functional cooperation of miR-125a, miR-125b, and miR-205 in entinostat-induced downregulation of erbB2/erbB3 and apoptosis in breast cancer cells. Cell Death Dis. 4, e556 (2013).

51. Liu CM, Wang RY, Saijilafu, Jiao ZX, Zhang BY, Zhou FQ. MicroRNA-138 and SIRT1 form a mutual negative feedback loop to regulate mammalian axon regeneration. Genes Dev. 27(13), 1473-1483 (2013).

52. Zhang $\mathrm{H}$, Zhang $\mathrm{H}$, Zhao $\mathrm{M}$ et al. MiR-138 inhibits tumor growth through repression of EZH2 in non-small cell lung cancer. Cell Physiol. Biochem. 31(1), 56-65 (2013).

53. Zhu Z, Tang J, Wang J, Duan G, Zhou L, Zhou X. MiR-138 acts as a tumor suppressor by targeting EZH2 and enhances cisplatin-induced apoptosis in osteosarcoma cells. PLoS ONE 11(3), e0150026 (2016).

54. Xu N, Papagiannakopoulos T, Pan G, Thomson JA, Kosik KS. MicroRNA-145 regulates OCT4, SOX2, and KLF4 and represses pluripotency in human embryonic stem cells. Cell 137(4), 647-658 (2009).

55. Xue G, Ren Z, Chen Y et al. A feedback regulation between miR-145 and DNA methyltransferase $3 \mathrm{~b}$ in prostate cancer cell and their responses to irradiation. Cancer Lett. 361(1), 121-127 (2015).

56. Suh SO, Chen Y, Zaman MS et al. MicroRNA-145 is regulated by DNA methylation and $\mathrm{p} 53$ gene mutation in prostate cancer. Carcinogenesis 32(5), 772-778 (2011).

57. Xia W, Chen Q, Wang J et al. DNA methylation mediated silencing of microRNA-145 is a potential prognostic marker in patients with lung adenocarcinoma. Sci. Rep. 5, 16901 (2015).

58. Juan AH, Kumar RM, Marx JG, Young RA, Sartorelli V. Mir-214-dependent regulation of the polycomb protein EZH2 in skeletal muscle and embryonic stem cells. Mol. Cell 36(1), 61-74 (2009).

-• First paper in which the epigenetic role of miR-214 was addressed and its involvement in a mutually negative feedback loop with EZH2 was observed. 